\title{
镉胁迫对姬松茸生长和镉吸收累积的影响
}

\author{
刘朋虎 ${ }^{1,3}$, 陈 华 ${ }^{2,3}{ }^{*}$, 李 波 $^{3}$, 王义祥 2,3 ,翁伯琦 ${ }^{2,3}$ \\ 1 福建农林大学国家菌草工程技术研究中心,福州 350002 \\ 2 福建省农业科学院农业生态研究所,福州 350013 \\ 3 福建省红壤山地农业生态过程重点实验室,福州 350013
}

摘要:利用外源镉添加的袋栽试验, 系统研究了镉对姬松茸 J37 和 J1 两菌株农艺性状、子实体产量、氨基酸和镉含量的影响, 以 求为低富集镉的姬松茸品种选育和安全栽培提供科学依据。结果表明外源镉添加对姬松茸 J37 和 J1 品种子实体的农艺性状 的影响各异, 两菌株单个子实体重量、子实体高度、菌盖直径、菌盖厚度和菌柄直径等均与外源镉浓度具有显著的负相关性, 其 中子实体重量与外源镉浓度间的相关性最大; $15 \mathrm{mg} / \mathrm{kg}$ 镉水平是两菌株农艺性状发生显著变化的敏感点。不同镉水平下 J37 菌株单个子实体重量比 $\mathrm{J} 1$ 菌株高 $10.9 \%$ - 36.6\%, 表现为较强镉耐受性。姬松茸 J37 与 J1 菌株子实体产量、氨基酸和镉含量均 随着潮次的增加而降低, 子实体产量和氨基酸含量随着外源镉水平增加而降低,而镉含量随外源镉水平增加而增加。与外源镉 $0 \mathrm{mg} / \mathrm{kg}$ 处理相比, 当外源镉水平达 $15 \mathrm{mg} / \mathrm{kg}$ 时, $\mathrm{J} 1$ 和 J37 子实体总产量分别下降 $23.4 \%$ 和 $13.4 \%$ 以上, $\mathrm{J} 1$ 和 $\mathrm{J} 37$ 子实体镉含量 分别提高 $51.5 \%$ 、 $13.7 \%$ 以上, 且 $\mathrm{J} 1$ 子实体镉含量已达到食品卫生标准的临界值。当外源镉水平达到 $35 \mathrm{mg} / \mathrm{kg}$ 时, J1 和 J37 子 实体产量分别下降 45\%、32\%以上, J1 和 J37 子实体镉含量分别提高 $75 \%$ 和 $68 \%$ 以上; 在 $0 、 15 、 35 \mathrm{mg} / \mathrm{kg}$ 镉水平下, J37 菌株四潮 子实体氨基酸含量平均比 $\mathrm{J} 1$ 菌株提高了 $20.1 \% 、 16.6 \%$ 和 $16.1 \%$, 且两菌株间的差异均达到显著性水平 $(P<0.05)$ 。随着姬松茸 收获潮次的增加, $\mathrm{J} 1$ 和 J37 菌株子实体氨基酸含量总体呈逐渐降低的趋势, 但潮次间的差异不显著。试验结果显示, 姬松茸品 种 J37 比 J1 相对耐受镉胁迫,但培养料中镉含量应控制在 $15 \mathrm{mg} / \mathrm{kg}$ 以下,才有助于保障子实体产品符合食品卫生标准。

关键词 : 姬松茸; 镉胁迫; 子实体; 农艺性状; 产量; 镉含量

\section{Effect of cadmium stress on the growth and cadmium absorption of Agaricus blazei Murill.}

\author{
LIU Penghu ${ }^{1,3}$, CHEN Hua ${ }^{2,3, *}$, LI Bo ${ }^{3}$, WANG Yixiang ${ }^{2,3}$, WENG Boqi ${ }^{2,3}$ \\ 1 National Engineering Research Center of Juncao Technology, Füjan Agriculture and Forestry University, Fuzhou 350002, China \\ 2 Agricultural Ecology Institute, Fujian Academy of Agricultural Sciences, Fuzhou 350013, China \\ 3 Fujian Key Laboratory of Agroecological Processes in Red Soil Hilly Region, Fuzhou 350013, China
}

\begin{abstract}
A bag cultivation experiment with exogenous cadmium was used to compare the differences in agronomic traits, yield, amino acid and cadmium content of Agaricus blazei strains J37 and J1, which could provide scientific basis for selection and cultivation of Agaricus blazei with low cadmium enrichment. The results showed that the addition of exogenous cadmium had different effects on the body agronomic characteristics of Agaricus blazei strains J37 and J1. The weight, height, cap diameter, cap thickness and stem diameter of individual body for J1 and J 37 had a significantly negative correlation with exogenous cadmium concentration. The correlation between body weight and exogenous cadmium concentration was the largest. The $15 \mathrm{mg} / \mathrm{kg}$ cadmium level was the sensitive point for the significant changes in the
\end{abstract}

基金项目:中央引导地方科技发展专项 (2020L3030); 福建省科技厅农业引导性(重点)项目(2020N0007); 福建省农科院创新团队项目

收稿日期:2020-05-12; 网络出版日期:2021-10-11

*通讯作者 Corresponding author.E-mail: fjch1976@163.com 
agronomic characteristics of them. The individual body weight of $\mathrm{J} 37$ was $10.9 \%-36.6 \%$ higher than that of J1 under different cadmium levels, which indicated that $\mathrm{J} 37$ had strong cadmium tolerance. The yield, amino acid and cadmium content of the bodies of J37 and J1 strains decreased with the increase of cultivation numbers. The yield and amino acid content of them decreased with the increase of exogenous cadmium level, but the cadmium content increased with the increase of exogenous cadmium level. When the exogenous cadmium level was higher than $15 \mathrm{mg} / \mathrm{kg}$, the yield of J1 and J37 decreased by $23.4 \%$ and $13.4 \%$ than that of no cadmium treatment, respectively. The cadmium content of them increased by $51.5 \%$ and $13.7 \%$, respectively, which of $\mathrm{J} 1$ was close to food hygiene standards. When the level of exogenous cadmium reached $35 \mathrm{mg} / \mathrm{kg}$, the yield of $\mathrm{J} 1$ and $\mathrm{J} 37$ decreased by $45 \%$ and $32 \%$, and the cadmium content of them increased by $75 \%$ and $68 \%$, respectively. The amino acid content in the fruit bodies of J37 with cadmium treatments ( 0,15 and $35 \mathrm{mg} / \mathrm{kg}$ ) increased by $20.1 \%, 16.6 \%$, and $16.1 \%$ than that of $\mathrm{J} 1$, respectively, and the difference between them reached a significant level $(P<0.05)$. With the increase of harvest tides, the amino acids contents of J1 and J37 gradually decreased, but the difference between the tides was not significant. The results showed that J37 was relatively resistant to cadmium stress than $\mathrm{J} 1$, but the cadmium content in the culture material should be controlled below $15 \mathrm{mg} / \mathrm{kg}$, which could help to ensure that the fruit body products meet the food hygiene standards.

Key Words : Agaricus blazei Murill; cadmium stress; sporocarp; agronomic trait; yield; cadmium content

姬松茸由于良好的品质与特殊的风味深受城乡消费者的喜爱 ${ }^{[1]}$ 。Cd 是土壤污染的主要因素之一,微量 的 $\mathrm{Cd}^{2+}$ 累积不仅对植物生长有毒害作用, 同时食品镉超标对人体健康产生较大的危害 ${ }^{[2-3]}$ 。姬松茸对镉等重 金属比较敏感,在生产栽培过程容易发生镉等重金属累积与超标现象,进而影响绿色生产与食品安全,引发人 们高度重视 ${ }^{[4-5]}$ 。有研究表明, 要避免与防控姬松茸镉污染或镉累积超标, 除了要着力选育低镉姬松茸品种 之外, 很重要的一个实践命题就是要深人探讨姬松茸吸收与富集镉的规律与主要影响因素, 尤其是深人了解 姬松咠在不同生长阶段对镉吸收与累积过程及其内在动态变化的关系 ${ }^{[6-7]}$, 着力探索姬松咠绿色高优栽培与 有效降低镉吸收累积的技术途径。本研究以项目组通过辐射而成功选育的低镉姬松茸品种 J37 与常规生产 品种姬松茸 $\mathrm{J} 1$ 为供试品种 ${ }^{[8]}$, 以外源添加不同浓度的镉为试验处理, 系统探索在不同镉浓度胁迫条件之下, 对姬松茸子实体生长及其镉含量变化的动态响应, 力求总结其内在变化规律, 为耐受栽培环节镉胁迫的姬松 茸品种选育及选用主要农艺性状作为镉毒害表征指标提供参考与借鉴, 也为进一步开展耐镉胁迫的姬松茸品 种分子生物学机制研究提供科学依据。

\section{1 材料与方法}

\section{1 试验材料}

姬松茸 (Agaricus blazei Murill.) 供试菌种 2 个, 包括姬松茸 J1 (常规生产品种) 与 J37 均由福建农林大学 国家菌草工程技术研究中心提供 ( 以下简称 $\mathrm{J} 1$ 与 J37)。姬松茸栽培的基本培养料配方为: 棉籽壳 $20 \%$,玉米 芯 $12.5 \%$,牛粪(干) $35 \%$,敖皮 $10 \%$,稻草(干) $20 \%, \mathrm{CaCO}_{3} 1.0 \%$, 石灰 $1.5 \%$ 。麦粒种: 小麦 $20 \mathrm{~kg}, \mathrm{CaSO}_{4} \cdot 2 \mathrm{H}_{2} \mathrm{O}$ $0.4 \mathrm{~kg}$ 。

\section{2 试验设计}

试验设 $0 、 5 、 15 、 25 、 35 、 45 、 55 、 65 \mathrm{mg} / \mathrm{kg}$ 共 8 个外源添加不同镉浓度处理, 将培养料按料水比 $1: 1.25$ 的比 例进行混合, 并将事先配制好的氯化镉母液按照试验设计适量加人培养料中。将搅拌均匀的培养料分装入聚 丙烯袋中,每袋装料 $1.8 \mathrm{~kg}$, 两个菌株的每个处理设 20 个重复,进行随机区组排列。

\section{3 栽培管理}

姬松茸的出菇栽培, 按照常规袋栽管理。先将分装好的栽培袋封口, 放人高压灭菌锅中, $121^{\circ} \mathrm{C}$ 灭菌 $2 \mathrm{~h}$; 随后在无菌接种箱中按照无菌操作接种, 再将接种好的菌袋放置在 $28{ }^{\circ} \mathrm{C}$ 恒温的培养室中; 待菌丝走满袋后, 
分别将栽培袋移到栽培室并按照随机区组排列, 开袋覆土。待出菇后分别采收, 称重, 烘干, 样品冷藏备用。

\section{4 子实体农艺性状及产量测定}

将采收后的新鲜子实体用小刀与小刷子轻轻去掉菌柄带出的土, 然后用天平和数显卡尺对子实体进行称 重和测量, 对每个菌株每个梯度的出菇数量、农艺性状、菇鲜重分别进行统计。农艺性状测量指标包括: 单个 子实体平均重量和高度、菌盖直径和厚度、菌柄直径。子实体农艺性状为第一潮菇 5 个重复数的测定平均值, 子实体产量分为四潮收获,每一潮产量测定分别为 3 个重复数的平均值。

\section{5 子实体中镉含量测定}

将新鲜子实体去土后, 放人 $60^{\circ} \mathrm{C}$ 条件下烘干至恒重,用高速粉碎机将烘干好的样品粉碎磨成粉。子实体 样品消解采用硝酸-过氧化氢混合微波消解。称取 $0.2 \mathrm{~g}$ 左右研磨过的子实体样品, 放人预先清洗干净的微 波消煮管中,加人 $5.0 \mathrm{~mL}$ 硝酸密封过夜; 第二天消煮前, 每个消煮管加人 $2.0 \mathrm{~mL}$ 过氧化氢, 在 $100{ }^{\circ} \mathrm{C}$ 预热 $25 \mathrm{~min}$; 之后将消煮管放置至常温后拧紧盖子, 放入微波消煮仪中进行消煮。将消煮好的液体淋洗到 $50 \mathrm{~mL}$ 容量瓶中, 并进行适当稀释,之后用火焰原子吸收光谱仪进行镉含量的测定 ${ }^{[9]}$ 。同时做空白试验, 每个样品 均设 3 个重复, 分析所用的试剂均为优级纯。在样品的测定过程中以木耳无机成分标准物质 (GBW 10089) 进 行分析质量控制, 结果显示标准物质测定数据较集中, 并且平均值接近标准值, 说明精密度高, 准确度也高, 符 合质控要求。

1.6 数据处理

采用 SPSS 19.0 数据处理软件对实验数据进行差异显著性分析和相关性分析。

\section{2 结果与分析}

2.1 镉胁迫对姬松咠子实体农艺性状的影响

很显然,农艺性状是农作物表现出的表征性状,其 可以代表作物本身品种特性 ${ }^{[10-11]}$ 。通过比较分析外源 镉胁迫下 J37、J1 两种姬松茸品种第一潮子实体的农艺 性状, 可以直观的反映添加不同浓度外源镉对姬松茸子 实体生长产生的动态影响。不同镉浓度胁迫处理对姬 松茸子实体农艺性状的影响结果见图 1一图 5。就单个 子实体重量而言(图 1), 两个姬松茸菌株单个子实体重 量随着外源镉浓度的增加呈下降的变化趋势。与镉水 平为 $0 \mathrm{mg} / \mathrm{kg}$ 处理相比, 镉胁迫处理 $\mathrm{J} 1$ 和 J37 菌株单个 子实体重量分别降低了 $5.4 \%-80.8 \%$ 和 $4.9 \%$ $77.1 \%$, 其中镉水平 $\geqslant 15 \mathrm{mg} / \mathrm{kg}$ 处理与 $0 \mathrm{mg} / \mathrm{kg}$ 处理间

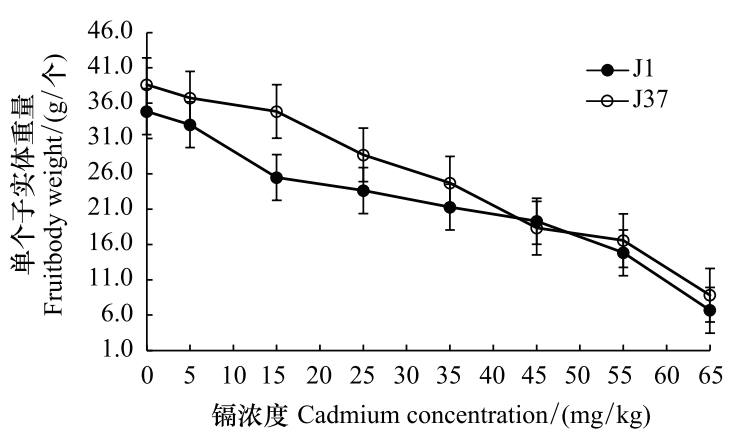

图 1 镉胁迫浓度对姬松茸子实体重量的影响

Fig. 1 Effect of cadmium stress on the fruitbody weight of Agaricus blazei

的差异均达到显著性水平 $(P<0.05)$ 。不同处理 J37 菌株单个子实体重量比 J1 菌株提高了 $10.9 \%$ - $36.6 \%$, 其 中 $15 \mathrm{mg} / \mathrm{kg}$ 镉处理两菌株间的差异达显著性水平 $(P<0.05)$, 其他处理两菌株间无显著性差异。就子实体高 度而言 (图 2), 两个姬松茸菌株随着外源镉浓度的增加呈降低的变化趋势。与镉水平为 $0 \mathrm{mg} / \mathrm{kg}$ 处理相比, 镉胁迫处理 $\mathrm{J} 1$ 和 $\mathrm{J} 37$ 菌株子实体高度分别降低了 $3.8 \%-75.1 \%$ 和 $3.9 \%-67.5 \%$, 其中镉水平 $\geqslant 15 \mathrm{mg} / \mathrm{kg}$ 处 理与 $0 \mathrm{mg} / \mathrm{kg}$ 处理间的差异均达到显著性水平 $(P<0.05)$ 。镉水平 $\geqslant 35 \mathrm{mg} / \mathrm{kg}$ 处理 $\mathrm{J} 37$ 菌株子实体高度比 $\mathrm{J} 1$ 菌株提高了 $1.1 \%-43.1 \%$,两菌株间的差异达显著性水平 $(P<0.05)$ 。

两个姬松茸菌株菌盖直径、菌盖厚度和菌柄直径亦均随着外源镉胁迫水平的提高呈减少的变化趋势 (图 $3 、 4$ 和 5)。就菌株而言, 不同镉胁迫处理 J37 菌株的菌盖直径、菌盖厚度和菌柄直径均大于 J1 菌株, 但两菌 株间无显著性差异。镉水平 $\geqslant 35 \mathrm{mg} / \mathrm{kg}$ 处理 $\mathrm{J} 1 、 \mathrm{~J} 37$ 菌株的菌盖直径、菌盖厚度和菌柄直径分别比镉水平为 $0 \mathrm{mg} / \mathrm{kg}$ 处理高出 $18.0 \%-80.9 \% 、 25.2 \%-71.4 \% 、 24.3 \%-79.6 \%$ 和 $17.7 \%-79.1 \% 、 23.0 \%-68.7 \% 、 17.9 \%-$ 
$78.2 \%$, 且镉水平 $\geqslant 35 \mathrm{mg} / \mathrm{kg}$ 处理与镉水平为 $0 \mathrm{mg} / \mathrm{kg}$ 处理间的差异均达到显著性水平 $(P<0.05)$ 。相关分析 表明 (表 1), 两菌株子实体重量、子实体高度、菌盖直径、菌盖厚度和菌柄直径与外源镉水平间均存在极显著 的负相关关系。

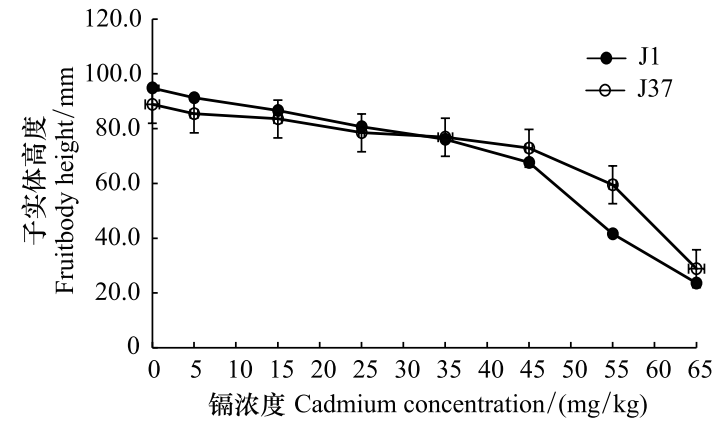

图 2 镉胁迫浓度对姬松茸子实体高度的影响

Fig.2 Effect of cadmium stress on the fruitbody height of Agaricus blazei

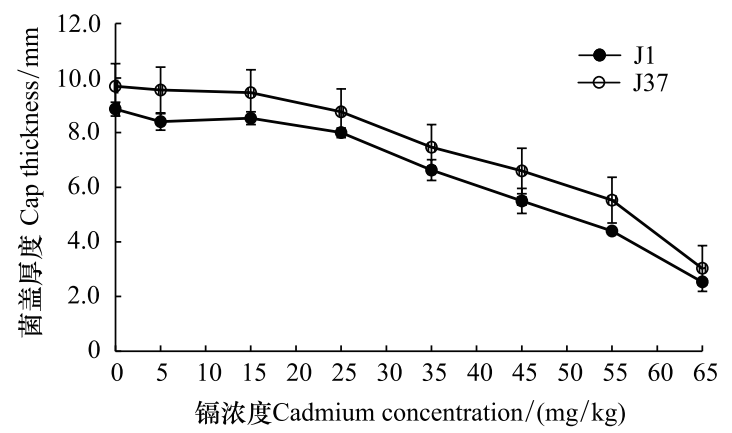

图 4 镉胁迫对姬松茸菌盖厚度的影响

Fig.4 Effect of cadmium stress on the cap thickness of Agaricus blazei

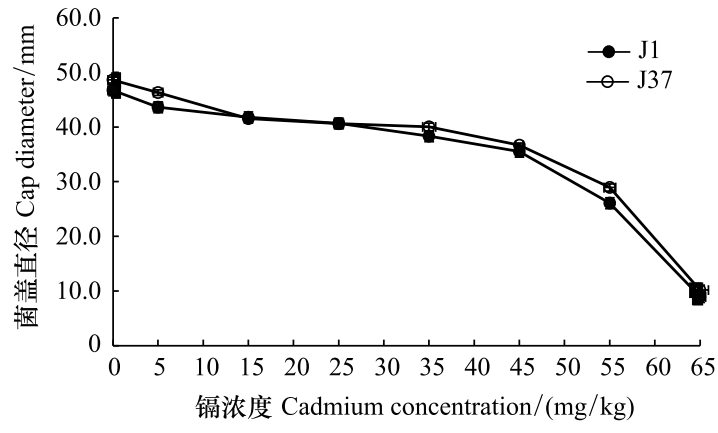

图 3 镉胁迫浓度对姬松茸菌盖直径的影响

Fig.3 Effect of cadmium stress on cap diameter of Agaricus blazei

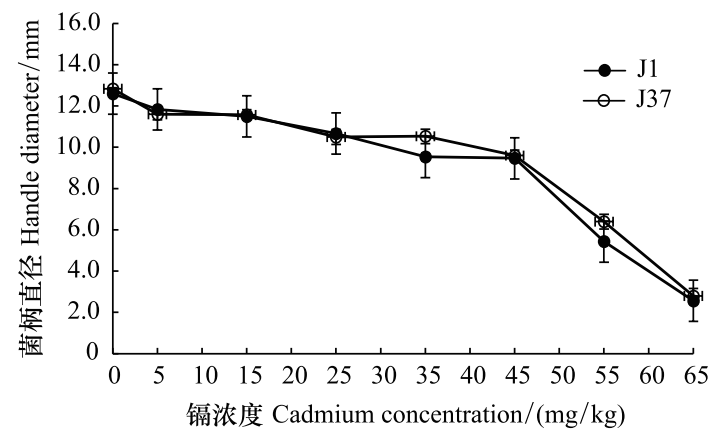

图 5 镉胁迫对姬松茸菌柄直径的影响

Fig.5 Effect of cadmium stress on the handle diameter of Agaricus blazei

表 1 姬松茸农艺性状与外源镉水平间的相关系数表

Table 1 Correlation coefficients between agronomic traits of Agaricus blazei and exogenous cadmium

\begin{tabular}{|c|c|c|c|c|c|}
\hline & $\begin{array}{c}\text { 子实体重量 } \\
\text { Fruit body weight }\end{array}$ & $\begin{array}{c}\text { 子实体高度 } \\
\text { Fruit body height }\end{array}$ & $\begin{array}{c}\text { 菌盖厚度 } \\
\text { Cap thickness }\end{array}$ & $\begin{array}{c}\text { 菌盖直径 } \\
\text { Cap diameter }\end{array}$ & $\begin{array}{c}\text { 菌柄直径 } \\
\text { Stem diameter }\end{array}$ \\
\hline 外源镉水平 Exogenous cadmium level & $-0.965 * *$ & $-0.907^{* *}$ & $-0.934 * *$ & $-0.890^{* *}$ & $-0.915^{* *}$ \\
\hline 子实体重量 Fruit body weight & 1 & $0.896^{* * *}$ & $0.953^{* *}$ & $0.906^{* * *}$ & $0.914^{* * *}$ \\
\hline 子实体高度 Fruit body height & $0.896^{* *}$ & 1 & $0.943^{* *}$ & $0.978^{* * *}$ & $0.985^{* *}$ \\
\hline 菌盖厚度 Cap thickness & $0.953^{* *}$ & $0.943^{* *}$ & 1 & $0.942^{* *}$ & $0.949^{* *}$ \\
\hline 菌盖直径 Cap diameter & $0.906^{* * *}$ & $0.978^{* * *}$ & $0.942^{* *}$ & 1 & $0.982^{* *}$ \\
\hline 菌柄直径 Stem diameter & $0.914^{* * *}$ & $0.985^{* *}$ & $0.949^{* *}$ & $0.982^{* *}$ & 1 \\
\hline
\end{tabular}

$* *$ 在 0.01 水平 (双侧) 上显著相关

2.2 镉胁迫对姬松茸子实体产量的影响

就 J1 与 J37 菌株相比, 在不添加外源镉的条件下, J37 菌株四潮菇子实体总产量比 J1 菌株增加了 $13.78 \%$, 且两菌株间的差异达显著性水平 $(P<0.05)$ 。镉水平为 $15 \mathrm{mg} / \mathrm{kg}$ 和 $35 \mathrm{mg} / \mathrm{kg}$ 条件下, $\mathrm{J} 37$ 四潮菇子 实体总产量分别比 J1 菌株高出 $28.2 \%$ 和 $40.9 \%$, 说明 J37 菌株比 J1 菌株对镉具有更高的耐受性。图 6 还显 
示, 随着潮次的增加,姬松茸两菌株子实体产量均呈逐渐降低的趋势。不同镉胁迫处理 $\mathrm{J} 1$ 菌株第一、二、三、 四潮子实体产量分别占总产量的 $39.8 \%-42.3 \% 、 23.9 \%-26.7 \% 、 20.0 \%-22.2 \% 、 11.0 \%-12.9 \%$; J37 菌株第 一、二、三、四潮子实体产量分别占总产量的 $36.7 \%-43.9 \% 、 23.4 \%-30.6 \% 、 19.8 \%-22.1 \% 、 11.6 \%-12.9 \%$ 。 就镉胁迫影响而言, $15 \mathrm{mg} / \mathrm{kg}$ 和 $35 \mathrm{mg} / \mathrm{kg}$ 镉胁迫处理 $\mathrm{J} 1$ 菌株子实体产量分别比未添加镉处理降低了 $23.4 \%$ 和 $45.4 \%, \mathrm{~J} 37$ 菌株分别降低了 $13.4 \%$ 和 $32.1 \%, 15 \mathrm{mg} / \mathrm{kg}$ 和 $35 \mathrm{mg} / \mathrm{kg}$ 与未添加镉处理间的差异均达到显著性 水平 $(P<0.05)$ 。

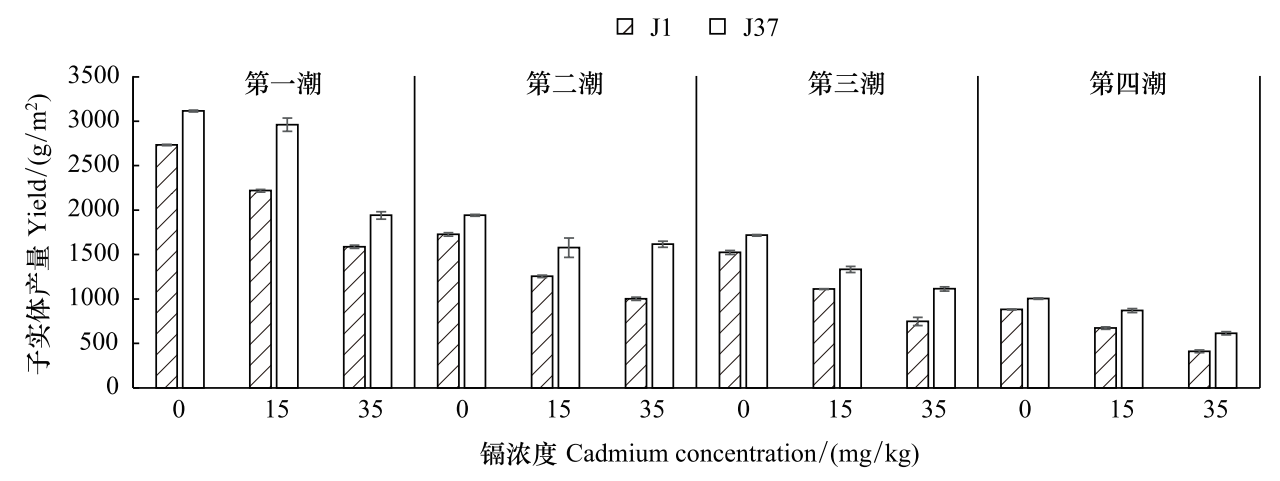

图 6 镉胁迫理对姬松茸子实体产量的影响

Fig.6 Effect of cadmium stress on fruitbody yield of Agaricus blazei

\section{3 镉胁迫对姬松茸子实体镉含量的影响}

$0 、 15 、 35 \mathrm{mg} / \mathrm{kg}$ 镉水平下, $\mathrm{J} 37$ 菌株四潮子实体镉含量平均比 J1 菌株降低了 $39.2 \% 、 56.0 \%$ 和 $31.3 \%$, 且两 菌株间的差异均达到显著性水平 $(P<0.05)$, 说明 $\mathrm{J} 37$ 比 $\mathrm{J} 1$ 菌株具有较低的镉富集特性。随着潮次的增加, J1 和 J37 菌株子实体镉含量均呈现逐渐降低的趋势 (图 7)。不同镉处理 J1 菌株第二、三、四潮菇子实体镉含量 分别比第一潮降低了 $6.2 \%-14.0 \% 、 18.8 \%-26.5 \% 、 26.0 \%-32.1 \%$; J37 菌株分别降低了 $8.1 \%-21.9 \%$ 、 $10.5 \%-32.7 \% 、 28.1 \%$ - $40.4 \%$ 。就镉胁迫影响而言, $15 \mathrm{mg} / \mathrm{kg}$ 和 $35 \mathrm{mg} / \mathrm{kg}$ 镉胁迫处理 J1 菌株四潮菇子实体 平均镉含量分别比 $0 \mathrm{mg} / \mathrm{kg}$ 镉胁迫处理增加了 $51.5 \%$ 和 $75.1 \%$; J37 菌株平均增加了 $13.7 \%$ 和 $68.7 \%, 15 \mathrm{mg} /$ $\mathrm{kg}$ 和 $35 \mathrm{mg} / \mathrm{kg}$ 与 $0 \mathrm{mg} / \mathrm{kg}$ 镉处理间的差异均达到显著性水平 $(P<0.05)$ 。当镉水平为 $15 \mathrm{mg} / \mathrm{kg}$ 时, J1 菌株子 实体镉含量已接近姬松咠镉卫生标准临界值 $(10 \mathrm{mg} / \mathrm{kg}$ 干品)。

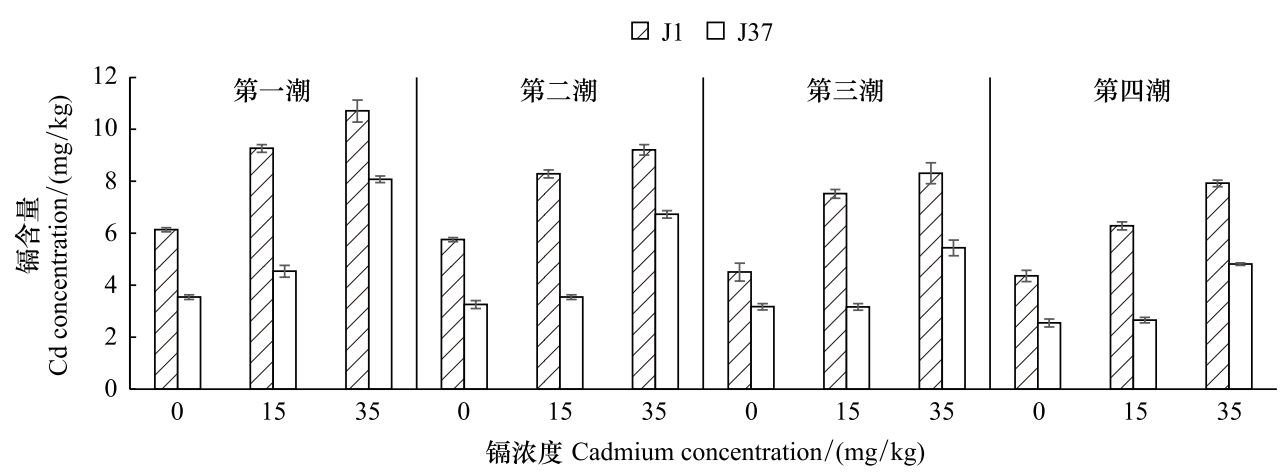

图 7 镉胁迫对姬松茸子实体镉含量的影响

Fig.7 Effect of cadmium stress on Cd concentration in the fruitbodies of Agaricus blazei

\section{4 镉胁迫对姬松茸子实体氨基酸含量的影响}

图 8 显示, $0 、 15$ 和 $35 \mathrm{mg} / \mathrm{kg}$ 镉水平下, $\mathrm{J} 37$ 菌株四潮子实体氨基酸含量平均比 $\mathrm{J} 1$ 菌株提高了 $20.1 \%$ 、 $16.6 \%$ 和 $16.1 \%$, 且两菌株间的差异均达到显著性水平 $(P<0.05)$ 。随着潮次的增加, $\mathrm{J} 1$ 和 $\mathrm{J} 37$ 菌株子实体氨 
基酸含量总体呈逐渐降低的趋势,但潮次间的差异不显著。不同镉处理 $\mathrm{J} 1$ 菌株第二、三、四潮菇子实体氨基 酸含量分别比第一潮降低了 $8.9 \%$ - $9.9 \% 、 10.4 \%-15.6 \% 、 10.3-19.3 \%, \mathrm{~J} 37$ 菌株分别降低了 $4.0 \%-5.4 \%$ 、 $8.7 \%-9.8 \% 、 13.2 \%-14.8 \%$ 。就镉胁迫影响而言, $15 \mathrm{mg} / \mathrm{kg}$ 和 $35 \mathrm{mg} / \mathrm{kg}$ 镉胁迫处理 $\mathrm{J} 1$ 菌株四潮菇子实体平 均氨基酸含量分别比 $0 \mathrm{mg} / \mathrm{kg}$ 镉胁迫处理降低了 $5.6 \%$ 和 $13.5 \%, \mathrm{~J} 37$ 菌株平均增加了 $7.9 \%$ 和 $16.4 \%$, 其中 $35 \mathrm{mg} / \mathrm{kg}$ 与 $0 \mathrm{mg} / \mathrm{kg}$ 镉处理间的差异达到显著性水平 $(P<0.05)$ 。

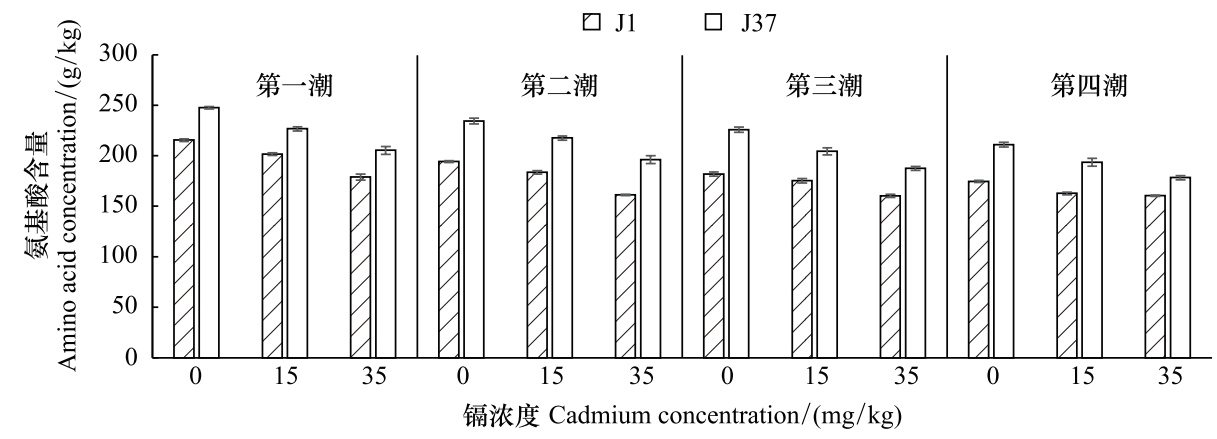

图 8 镉胁迫对姬松茸子实体氨基酸含量的影响

Fig.8 Effect of cadmium stress on amino acid concentration in the fruitbodies of Agaricus blazei

\section{3 讨论}

从本试验结果显示, 单个子实体重量与子实体高度、菌盖直径、菌盖厚度、菌柄直径为从属关系, 因此它们 间的趋势变化相似。将外源镉水平与两菌株子实体农艺性状进行相关性分析, 结果表明, J1 与 J37 子实体各 农艺性状与外源镉水平之间均呈极显著负相关。随着外源镉水平的增加,两个菌株子实体生长明显受到镉的 抑制作用, 各生长指标均呈下降趋势; 且 $\mathrm{J} 1$ 子实体各农艺性状降低程度均大于 $\mathrm{J} 37$ 子实体, 说明 $\mathrm{J} 1$ 子实体生 长受到镉的抑制程度大于 $\mathrm{J} 37$ 子实体。作为直观的判断指标应该为单个子实体重量, 其是生产实践的关键要 素,可以供生产管理者参考借鉴。

姬松茸生长过程对镉较为敏感已为生产实践所证实 ${ }^{[12-14]}$, 要避免姬松茸镉污染或者镉超标所造成危害 与损失, 除了着力选育低镉姬松茸新品种之外 ${ }^{[15]}$, 还需要深人探讨姬松茸生长过程镉吸收与富集机理, 尤其 是阐明影响姬松茸生长势——镉危害之间的内在关系及其动态响应具有重要参考价值 ${ }^{[16-18]}$ 。本试验研究表 明,外源镉水平 $<15 \mathrm{mg} / \mathrm{kg}$ 对姬松茸农艺性状、产量的影响不显著, 但当镉水平 $\geqslant 15 \mathrm{mg} / \mathrm{kg}$ 时, 两菌株农艺性 状发生显著变化,产量下降了 $13.4 \%$ 以上,镉含量显著增加且已临界食品卫生标准,这或许是镉元素对姬松茸 菌丝、子实体细胞的早期刺激效应所引发的短期行为,待镉元素吸收并逐步累积,其对农艺性状以及产量与镉 含量的影响就很快显现出来 ${ }^{[19]}$, 进而需要进一步探讨镉胁迫对细胞结构的影响 ${ }^{[20-21]}$, 同时需要研究镉胁迫 对姬松茸生长过程酶系统动态变化的影响 ${ }^{[22-24]}$, 力求进行多因素相关分析, 进而有目的或者有针对性地开展 分子生物学研究, 力求从基因组水平阐明内在关系 ${ }^{[25]}$, 并确定诊断指标, 为姬松茸高产优质育种提供科学 依据。

\section{4 结论}

不同镉胁迫浓度处理对姬松茸 J37 和 J1 子实体的农艺性状的影响各异。总体趋势是随着外源镉添加浓 度的增加, 其单个子实体重量、子实体高度、菌盖直径、菌盖厚度、菌柄直径等 5 项指标呈降低的趋势; 单个子 实体重量与外源镉水平间相关性最高。J37 与 $\mathrm{J} 1$ 菌株农艺性状变化的镉胁迫临界点均为 $15 \mathrm{mg} / \mathrm{kg}$; J37 菌株 耐受镉胁迫能力优于 $\mathrm{J} 1$ 菌株。

姬松茸 $\mathrm{J} 37$ 与 $\mathrm{J} 1$ 菌株产量、子实体中氨基酸和镉含量均随着潮次的增加而降低, 子实体产量和氨基酸含 
量随着外源镉水平增加而降低, 子实体镉含量随镉水平增加而增加。当外源镉水平高于 $15 \mathrm{mg} / \mathrm{kg}$ 时, $\mathrm{J} 1$ 和 $\mathrm{J} 37$ 子实体产量分别下降 $23.4 \%$ 和 $13.4 \%$ 以上, 镉含量分别提高 $51.5 \%$ 和 $13.7 \% \%$ 以上,且临界食品卫生标准。 由此认为姬松茸栽培基质镉浓度要控制在 $15 \mathrm{mg} / \mathrm{kg}$ 以内, 是防控姬松咠生产过程镉污染或者镉超标关键点。 除了选择耐镉危害及其能阻控镉吸收累积的品种之外,还在于低镉栽培原料与覆盖土壤选择,力求从源头上 实施姬松茸的质量安全防控与绿色产品生产。

\section{参考文献( References)：}

[ 1 ] 林杰, 蔡丹风, 王雪英. 食用菌珍品——姬松茸. 福建农业, 1995, (8): 10-10.

[ 2 ] 七良朋, 王祖伟. 滨海盐渍土壤中不同类型盐生植物富集镉的效应. 生态学报, 2017, 37(14)：4656-4662.

[ 3 ] 魏晓, 张鹏博, 赵丹丹, Bocharnikova E, Matichenkov V, Dmitry D. 水稻土施硅对土壤-水稻系统中镉的降低效果. 生态学报, 2018, 38 (5) : 1600-1606.

[ 4 ] 李三暑, 江枝和, 颜明娟, 郑永标, 黄乃江, 杨佩玉. 福建省姬松茸菇镉污染状况及其防治. 江西农业大学学报, 2000, 22 (1): 94-97.

[ 5 ] 刘朋虎, 赖瑞联, 陈华, 王义祥, 翁伯琦. 镉对食用菌生长的影响及防控技术研究进展. 生态环境学报, 2019, 28(2): 419-428.

[ 6 ] 刘朋虎, 李波, 江枝和, 王义祥, 翁伯琦. 姬松茸菌株 $\mathrm{J}_{1}$ 与 $\mathrm{J}_{77}$ 镉富集差异及生理响应机制. 农业环境科学学报, 2017, 36(5) : 863-868.

[ 7 ] 刘朋虎, 李波, 江枝和, 雷锦桂, 翁伯琦, 黄在兴. 镉对姬松茸农艺性状及矿物质元素吸收的影响. 农业环境科学学报, 2018, 37(1): 58-63.

[ 8 ] 刘朋虎, 陈爱华, 江枝和, 雷锦桂, 王义祥, 翁伯琦. 姬松茸“福姬 $J_{77}$ ”新菌株选育研究. 福建农业学报, 2012, 27 (12): 1333-1338.

[ 9 ] 李春香, 韩娟, 徐小慧, 侯延民. 乙醇-硫酸铵双水相萃取-火焰原子吸收光谱法测定镉. 治金分析, 2009, 29(9) : 60-65.

[10] 张焕裕. 作物农艺性状整齐度的研究进展. 湖南农业科学, 2005, (4) : 33-36.

[11] 刘娜, 张敏, 肖千明, 肖军, 肇莹. 不同蛹虫草菌株农艺性状及有效成分含量比较. 园艺与种苗, 2018, 38(2) : 28-31.

[12] 黄建成, 李开本, 何锦星, 余应瑞, 林衍铨. 姬松茸镉累积特性研究 I. 培养料镉污染对子实体的效应. 福建农业学报, 2006, 21 (3): 240-243.

[13] 徐丽红, 何莎莉, 吴应沝, 叶长文, 张永志, 王钢军. 姬松茸对有害重金属镉的吸收富集规律及控制技术研究. 中国食品学报, 2010, 10 (4) : 152-158.

[14] 林戊斌. 镉在姬松苜生产中迁移、分布规律及降低镉含量措施的研究 [D]. 福州: 福建农林大学, 2011.

[15] 李波. 镉胁迫下两个姬松茸品种生长及镉富集特性研究 [D]. 福州: 福建农林大学, 2016.

[16] 施巧琴, 林琳, 陈哲超, 陈松生, 谢必峰, 吴松刚, 陈静仪, 柯毅龙, 李贤明, 林园. 重金属在食用菌中的富集及对其生长代谢的影响. 菌 物学报, $1991,10(4): 301-311$.

[17] 霍存录, 商圆圆, 竹文坤, 贺新生. 红平菇 (Pleurotus djamor) 的镉耐性及其对镉去除能力的研究. 农业环境科学学报, 2013, 32 (5) : 966-971.

[18］黄敏敏, 江枝和, 翁伯琦, 王义祥. 镉对姬松茸菌丝体细胞超微结构的影响. 热带作物学报, 2011, 32(6)：1082-1085.

[19] Melgar M J, Alonso J, Pérez-López M, García M A. Influence of some factors in toxicity and accumulation of cadmium from edible wild macrofungi in NW Spain. Journal of Environmental Science and Health, Part B, 1998,33(4) : 439-455.

[20] 徐丽红, 吴应沝, 陈俏彪, 叶长文, 王钢军, 张永志. 香菇 (Lentinus edodes) 对重金属镉 (Cd) 的吸收规律及控制技术研究. 农业环境科学 学报, $2011,30(7): 1300-1304$.

[21] 黄建成, 余应瑞, 应正河, 李开本. 姬松茸镉累积特性研究: II 镉胁迫对菌丝及子实体生长发育的影响. 农业环境科学学报, 2008,27 (1) : 78-81.

[22] Cobbett C, Goldsbrough P. Phytochelatins and metallothioneins: roles in heavy metal detoxification and homeostasis. Annual Review of Plant Biology, 2002, 53: 159-182.

[23] Romero-Isart N, Vašák M. Advances in the structure and chemistry of metallothioneins. Journal of Inorganic Biochemistry, 2002, 88 (3/4): 388-396.

[24] 李三暑, 雷锦桂, 陈惠成. 镉、磷、钻在姬松茸细胞内的积累和分布特征及其交互作用. 食用菌学报, 2001, 8(4): 24-27.

[25] 刘高否. 中国香菇重要性状的全基因组关联分析 [D]. 武汉: 华中农业大学, 2017. 